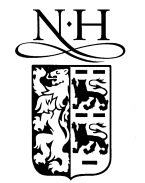

ELSEVIER

\title{
Dissipative dynamics of an open Bose Einstein condensate
}

\author{
F.T. Arecchi ${ }^{1}$, J. Bragard ${ }^{*, 2}$, L.M. Castellano ${ }^{3}$ \\ Istituto Nazionale di Ottica, Largo E. Fermi, 6, I-50125 Florence, Italy
}

Received 20 August 1999; received in revised form 27 October 1999; accepted 2 November 1999

\begin{abstract}
As an atomic Bose Einstein condensate (BEC) is coupled to a source of uncondensed atoms at the same temperature and to a sink (extraction towards an atom laser) the idealized description in terms of a Gross-Pitaevsky equation (GP) no longer holds. Under suitable physical assumptions we show that the dissipative BEC obeys a Complex Ginzburg Landau equation (CGL) and for some parameter range it undergoes a space time patterning. As a consequence, the density of BEC atoms within the trap displays non trivial space time correlations, which can be detected by monitoring the density profile of the outgoing atom laser. The patterning condition requires a negative scattering length, as e.g. in ${ }^{7} \mathrm{Li}$. In such a case we expect a many domain collapsed regime, rather than a single one as reported for a closed BEC. C) 2000 Elsevier Science B.V. All rights reserved.
\end{abstract}

\section{Introduction}

The BEC dynamics in an atomic trap is ruled by a GP [1,2] which in fact is a nonlinear Schrödinger equation (NLS) describing a conservative motion. Experimental evidence of BEC in a trap [3-5] confirmed qualitatively a dynamical picture based on a GP description. On the other hand, extraction of BEC-atoms toward an atom laser [6-8] introduces a dissipation which must be compensated for by a transfer from the uncondensed fraction of trapped atoms. Those ones on their turn must be refilled by a

\footnotetext{
* Corresponding author. E-mail: jbragard@ino.it

${ }^{1}$ Also at Dept. of Physics, University of Florence, Florence, Italy. E-mail: arecchi@ino.it

${ }^{2}$ Also at Dept. of Physics, University of Liege, Liege, Belgium.

${ }^{3}$ On leave from Dept. of Physics, University of Antioquia, Medellin, Colombia.
}

pumping process which, in the actual laboratory set ups is a discontinuous process [6-8] but that we here consider as a continuous refilling, even though no working scheme is available yet. In Section 2 we describe the addition of dissipative interactions through coupled rate equations, as done by Kneer et al. [9]. In Section 3 we provide the physical grounds for an additional space dependent (diffusive) process and introduce an adiabatic elimination procedure, whereby we arrive at a closed equation which in fact is a CGL. In Section 4 we rescale the CGL around threshold for both positive $\left({ }^{87} \mathrm{Rb}\right)$ and negative $\left({ }^{7} \mathrm{Li}\right)$ scattering lengths, showing that in the first case the $\mathrm{BEC}$ is stable against space time variations, whereas in the second case the system can easily cross the instability barrier (so-called Benjamin-Feir line [10 12]). In Section 5 we present numerical results showing that in the unstable case, rather than collapsing into one singular spot as in the isolated BEC, the open system presents many uncorrelated domains 
(space-time chaos). In Section 6 we compare the strength of the nonlinear dissipative term introduced by us with the 3-body recombination rate.

\section{The dynamics of an open BEC}

We know $[1,2]$ that a BEC is modeled by the GP $\mathrm{i} \hbar \frac{\partial \phi}{\partial t}=-\frac{\hbar^{2}}{2 m} \nabla^{2} \phi+V_{\mathrm{ext}} \phi+g|\phi|^{2} \phi$.

where $\phi=\sqrt{\rho} \mathrm{e}^{\mathrm{i} \theta}$ is the macroscopic wave function describing the probability amplitude of the condensate, $V_{\mathrm{ext}}$ is the trap potential, shaped as a harmonic oscillator with frequency $\omega$, and $g$ is the coupling constant for the nonlinear (density $|\phi|^{2}$ dependent) self interaction. $g$ is proportional to the $s$-wave scattering length $a_{s}$

$g=\frac{4 \pi \hbar^{2}}{m} a_{s}$.

We discuss specific experimental situations concerning ${ }^{87} \mathrm{Rb}$ atoms $\left(m=1.4 \times 10^{-25} \mathrm{~kg}, a_{s}=5.77\right.$ $\mathrm{nm})$ and ${ }^{7} \mathrm{Li}$ atoms $\left(m=0.115 \times 10^{-25} \mathrm{~kg}, a_{s}=\right.$ $-1.45 \mathrm{~nm}$ ) [2]. For an anisotropic trap the frequency is $\omega=\left(\omega_{x} \omega_{y} \omega_{z}\right)^{1 / 3}[2-5]$. The above equation is formally a conservative non linear Schrödinger equation (NLS). Thus, it is a straightforward task to attach to a BEC all those space-time features familiar of a NLS as e.g. solitary structures and vortices $[13,14]$, which have been explored in the recent past for a NLS, mainly in connection with pulse propagation in optical fibers $[15,16]$.

On the other hand the idealized picture of a BEC in an isolated system is in contrast with two physical facts, namely,

i) The BEC is made of that fraction of atoms which have collapsed into the ground state $(n=0)$ of the harmonic oscillator trap potential; these atoms interact via collisions with those ones which are distributed over the excited states $(n>0)$ of the trap. The uncondensed atomic density $n_{u}$ evolves in time not only because of the coupling with the condensed phase described by $\phi$, but also because trapping and cooling processes imply a feeding (pumping) at a local rate $R(\boldsymbol{r})$, the space dependence accounts for the non uniformities of the pumping process as well as for losses due to escape from the trap, at a rate $\gamma_{u}$. ii) In order to have an atom laser, a radio frequency (rf) field is applied to the trap. The rf changes the magnetic quantum number of the atoms' ground state, thus transforming the trapping potential into a repulsive one and letting atoms escape from the BEC at a rate $\gamma_{c}[6-8]$.

Both i) and ii) have been modeled by Kneer et al. [9] by adding dissipative terms to Eq. (1) and coupling the resulting equation with a rate equation for $n_{u}$ (as a fact, rate equation coupling between condensed and uncondensed atoms had already been introduced by Speew et al. [17]). In a slightly different formulation, this amounts to the following equations

$$
\begin{aligned}
\mathrm{i} \hbar \frac{\partial \phi}{\partial t}= & -\frac{\hbar^{2}}{2 m} \nabla^{2} \phi+V_{\mathrm{ext}} \phi \\
& +g|\phi|^{2} \phi-\frac{\mathrm{i}}{2} \hbar \gamma_{c} \phi+\frac{\mathrm{i}}{2} \hbar \Gamma n_{u} \phi
\end{aligned}
$$

and

$n_{\dot{u}}=R(\boldsymbol{r})-\gamma_{u} n_{u}-\Gamma n_{u} n_{c}$.

here $\Gamma$ is the rate constant coupling the condensed field $\phi$ with the uncondensed density $n_{u}$, and $n_{c}=1$ $\left.\phi\right|^{2}$ is the local density of the condensed phase. We have modified the model of Ref. [9] as follows. At variance with [9], where Eqs. (3) and (4) were written for the overall atomic population $N_{u}$ over the whole trap volume $\mathrm{V}$, that is,

$N_{u}=\int n_{u} \mathrm{~d}^{3} r$

which is then coupled to

$N_{c}=\int n_{c} \mathrm{~d}^{3} r=\int|\phi|^{2}(r) \mathrm{d}^{3} r$,

here we prefer to deal with a local coupling. In fact Eqs. (3) and (4) as written above are more convenient, as they refer to a local interaction. The coupling rate of uncondensed to condensed atoms, $\Gamma$ $\left(\mathrm{m}^{3} \mathrm{~s}^{-1}\right)$, is given by the global rate used in [9] which we call $\Gamma^{\prime}$, dividing by the trap volume $V$

$\Gamma^{\prime}=\frac{\Gamma}{V}\left(\mathrm{~s}^{-1}\right)$. 
Furthermore our local feeding rate $R(r)$ is related to the overall rate $R_{u}$ of [9] by

$$
R_{u}=\int R(r) \mathrm{d}^{3} r
$$

\section{CGL picture of the open BEC}

Eqs. (3) and (4) were the basis of the model reported in Ref. [9]. We wish to improve that picture, based on the following considerations. The uncondensed phase, $n_{u}$, is fed by a pumping process $R(r)$ which is in general non uniform, and is locally depleted by its coupling with the condensed phase. As a result, $n_{u}$ has a sensible space dependence and hence it undergoes diffusion processes. Precisely, by the fluctuation-dissipation theorem $[18,19]$, the diffusion in velocity is given by

$D_{v}=\gamma_{u} \frac{k_{B} T}{m}\left(\mathrm{~m}^{2} \mathrm{~s}^{-3}\right)$.

The corresponding diffusion constant in real space will be

$D_{r}=\frac{D_{v}}{\gamma_{u}^{2}}=\frac{1}{\gamma_{u}} \frac{k_{B} T}{m}\left(\mathrm{~m}^{2} \mathrm{~s}^{-1}\right)$.

For ${ }^{87} \mathrm{Rb}$ at $T=100 \mathrm{nK}$, and for $\gamma_{u}=5 \times 10^{2}$ $\mathrm{s}^{-1}$ (of the order of the average trap frequency $\tilde{\omega} \approx 415 \mathrm{~Hz}$ ) [9] this yields

$D_{r}=2 \times 10^{-8}\left(\mathrm{~m}^{2} \mathrm{~s}^{-1}\right)$.

Thus we must add the term $D_{r} \nabla^{2} n_{u}$ to Eq. (4). Once the BEC has been formed, the escape rate $\gamma_{c}$ in Eq. (3) is compensated for by the feeding rate $\Gamma n_{u}$. As we set the BEC close to threshold, because of critical slowing down, the $\phi$ dynamics will be much slower than the $n_{u}$ dynamics, thus we can apply an adiabatic elimination procedure [20], find a quasi stationary solution for $n_{u}$ in terms of $\phi$ and replace it into Eq. (3) which then becomes a closed equation for $\phi$.

We specify the above procedure by the following steps. First, rewrite Eq. (4) including diffusion

$n_{\dot{u}}=R(r)-\gamma_{u} n_{u}+D_{r} \nabla^{2} n_{u}-\Gamma n_{u}|\phi|^{2}$.

Next, we take its space Fourier transform. The linear terms are trivial, whereas the nonlinear term should provide a convolution integral. Even though the condensate is not uniform, we consider only the $k=0$ component in the nonlinear term which is just a perturbation; then its Fourier transform is $\mathscr{F} \mid \phi(r)$ $\left.\right|^{2}=|\phi|_{0}^{2} \delta(k)$ and Eq. (12) transforms as

$n_{\dot{k}}=R_{k}-\left(\gamma_{u}+D_{r} k^{2}\right) n_{k}-\Gamma n_{k}|\phi|_{0}^{2}$.

where $n_{k}=\mathscr{F} n_{u}, R_{k}=\mathscr{F} R(r)$. The adiabatic elimination procedure consists in taking the stationary solution of Eq. (13) and replacing into Eq. (3). The stationary solution of Eq. (13) is

$n_{k}=\frac{\frac{R_{k}}{\gamma_{u}}}{1+\frac{D_{r}}{\gamma_{u}} k^{2}+\frac{\Gamma}{\gamma_{u}}|\phi|^{2}}$.

For long wavelength perturbations and far from saturation, the two additional terms in the denominator are less than unity. Here we consider a cylindrical volume with $L_{x}=L_{y}=5 \mu \mathrm{m}, L_{z}=10 \mu \mathrm{m}(V=250$ $\left.(\mu \mathrm{m})^{3}\right)$ containing a condensate of $N_{c}=5 \times 10^{4}$ atoms at a temperature $T=100 \mathrm{nK}$ [21]. It follows that $k \approx O(1 / L) \approx 0.5 \times 10^{5} \mathrm{~m}^{-1}$, and hence $D_{r} k^{2} / \gamma_{u}<1$. We can then expand Eq. (14) as

$n_{k} \approx \frac{R_{k}}{\gamma_{u}}\left(1-\frac{D_{r}}{\gamma_{u}} k^{2}-\frac{\Gamma}{\gamma_{u}}|\phi|_{0}^{2}\right)$.

The inverse Fourier transform of Eq. (15) is an operator relation as

$n_{u}=\frac{R}{\gamma_{u}}\left(1+\frac{D_{r}}{\gamma_{u}} \nabla^{2}-\frac{\Gamma}{\gamma_{u}}|\phi|^{2}\right)$.

As we replace this expression into Eq. (3), the operator $\nabla^{2}$ acts on its right upon the space function $\phi$. By doing this, we derive at a closed equation for $\phi$ which reads as

$$
\begin{aligned}
\frac{\partial \phi}{\partial t}= & -\frac{\mathrm{i}}{\hbar}[G P]+\frac{1}{2}\left(\frac{R \Gamma}{\gamma_{u}}-\gamma_{c}\right) \phi+\frac{R D_{r} \Gamma}{2 \gamma_{u}^{2}} \nabla^{2} \phi \\
& -\frac{R \Gamma^{2}}{2 \gamma_{u}^{2}}|\phi|^{2} \phi,
\end{aligned}
$$


where the square brackets contain the right hand side of Eq. (1). We now write explicitly the GP terms thus arriving at the following CGL

$$
\begin{aligned}
\dot{\phi}= & {\left[\frac{1}{2}\left(\frac{R \Gamma}{\gamma_{u}}-\gamma_{c}\right)-\frac{\mathrm{i} V_{\text {ext }}}{\hbar}\right] \phi } \\
& +\left(\frac{R D_{r} \Gamma}{2 \gamma_{u}^{2}}+\frac{\mathrm{i} \hbar}{2 m}\right) \nabla^{2} \phi \\
& -\left(\frac{R \Gamma^{2}}{2 \gamma_{u}^{2}}+\mathrm{i} \frac{g}{\hbar}\right)|\phi|^{2} \phi .
\end{aligned}
$$

The dissipative terms of Eq. (18) represent respectively: i) difference between gain and losses, which implies a threshold condition; ii) a real diffusion which implies a spread of any local perturbation; iii) a real saturation term which provides a density dependent gain saturation.

\section{Rescaled CGL near threshold}

We herewith list the numerical values as taken from the experiment [21] or from Ref. [9]. We refer to a trap volume $V=0.25 \times 10^{-15} \mathrm{~m}^{3}$; with a loss rate $\gamma_{u}=500 \mathrm{~s}^{-1}$. If we take the value [9] $\Gamma^{\prime} \approx 7$ $\mathrm{s}^{-1}$, then $\Gamma=\Gamma^{\prime} V \approx 2 \times 10^{-15} \mathrm{~m}^{3} \mathrm{~s}^{-1}$. Furthermore, a reasonable estimate for the BEC escape rate toward the atom laser is [9] $\gamma_{c} \approx 50 \mathrm{~s}^{-1}$. Therefore the threshold condition (gain $=$ losses) is fulfilled for $R=\bar{R}$, where

$\bar{R} \Gamma / \gamma_{u}=\gamma_{c}$

here $\bar{R} \approx 12 \times 10^{18} \mathrm{~m}^{-3} \mathrm{~s}^{-1}$, corresponding to $R_{\bar{u}}=$ $\bar{R} V \approx 3000 \mathrm{~s}^{-1}$. Finally we have for ${ }^{87} \mathrm{Rb}[2] \mathrm{g} / \hbar=$ $\left(4 \pi \hbar a_{s}\right) / m=0.48 \times 10^{-16} \mathrm{~m}^{3} \mathrm{~s}^{-1}$. Furthermore, the treatment here outlined, with the cubic approximation (Eqs. (14) and (15)) requires $\left(|\phi|^{2} \Gamma\right) / \gamma_{u} \ll 1$, which holds for the $\mathrm{Rb}$ condensate up to $N_{c} \approx 100$.

We can now write the parametrized CGL Eq. (18) in the dimensionless form

$\dot{\phi}=\epsilon \phi+\left(1+\mathrm{i} c_{1}\right) \nabla^{2} \phi-\left(1+\mathrm{i} c_{2}\right)|\phi|^{2} \phi$.

where we have used the dimensionless time

$\tau=\gamma_{c} t$

and the dimensionless space coordinates

$$
\frac{x}{l_{o}}, \frac{y}{l_{o}}, \frac{z}{l_{o}},
$$

where

$l_{o}=\left(\frac{\bar{R} D_{r} \Gamma}{2 \gamma_{u}^{2} \gamma_{c}}\right)^{1 / 2} \approx 3 \times 10^{-6} \mathrm{~m}$

is the characteristic length associated to the CGL dissipative dynamics, and the dimensionless condensated wave-function

$|\tilde{\phi}|^{2}=\frac{\bar{R} \Gamma^{2}}{2 \gamma_{u}^{2} \gamma_{c}}|\phi|^{2}$.

Note that tilde has been dropped in Eq. (20). It follows from Eq. (18) that

$$
\begin{aligned}
& \epsilon=\frac{1}{2}\left(\frac{R \Gamma}{\gamma_{u} \gamma_{c}}-1\right)=\frac{1}{2}\left(\frac{R}{\bar{R}}-1\right), \\
& c_{1}=\frac{\hbar \gamma_{u}^{2}}{m R D_{r} \Gamma}, \\
& c_{2}=\frac{2 g \gamma_{u}^{2}}{\hbar R \Gamma^{2}},
\end{aligned}
$$

are the significant parameters of Eq. (20). They are pure numbers. The term $-\mathrm{i} V_{\text {ext }} / \hbar \phi=-\mathrm{i} c_{0} \phi$ in Eq. (18) can be eliminated by a rotation transformation $\phi \rightarrow \phi \mathrm{e}^{-\mathrm{i} c_{0} t}$.

We notice that Eq. (20), derived by sound physical assumptions, is far from being a purely conservative (GP) or purely dissipative (real Ginzburg Landau) equation, but it displays both characters.

However the Benjamin-Feir instability condition [10]

$c_{1}\left(-c_{2}\right)>1$

is not met by ${ }^{87} \mathrm{Rb}\left(c_{1}=0.52, c_{2}=2.05\right)$ and its dissipative CGL is fully inside the stable region. Hence the addition of dissipative terms may add interesting transient effects but does not lead to substantial qualitative changes with respect to the GP equation. Quite different is the case of ${ }^{7} \mathrm{Li}\left(c_{1}=0.04\right.$, $\left.c_{2}=-0.51\right)$. Indeed even though the values of $c_{1}$ and $c_{2}$ just listed give a stable dynamics, the fact that the scattering length is negative may lead to an instability if the parameters of the open BEC are slightly changed, e.g. if $\gamma_{c}$ is reduced by a factor 10 (which physically corresponds to a $\mathrm{Li}$ atom-laser with weaker losses). In such a case, we get $c_{1}=0.4$, 
$c_{2}=-5.1$ and the open $\mathrm{BEC}$ is in the unstable region; we will denote this experimental situation by referring to an open $\mathrm{Li}^{*}$ BEC.

\section{Numerical simulations}

As we have shown in Section 4, the coefficients of the CGL depend on the nature of the atoms forming the open BEC and also depend on the characteristic working parameters of the open BEC. Let us discuss the space time dynamics of the density of the condensed phase $|\phi|^{2}$. To do this, we proceed to the numerical integration of Eq. (20). The integration is performed on a two dimensional domain. This corresponds to a cross section of the 3-D cigar shape where the condensation takes place. This is justified by the fact that $\omega_{z} \ll \omega_{x}, \omega_{y}$. The simulations are done on a $200 \times 200(\mathrm{Rb})$ or $256 \times 256$ $\left(\mathrm{Li}^{*}\right)$ grid starting with an initial Gaussian distribution at the center of the domain. The numerical integration code is based on a semi-implicit scheme

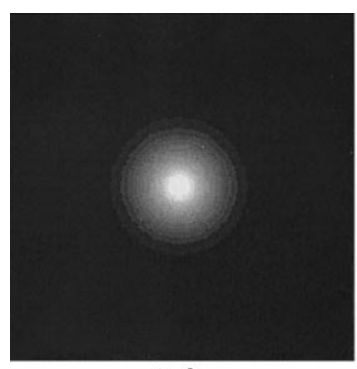

$\mathrm{t}=0$

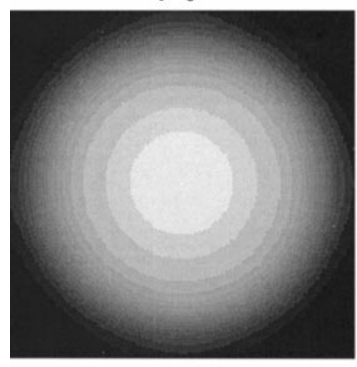

$\mathrm{t}=10$

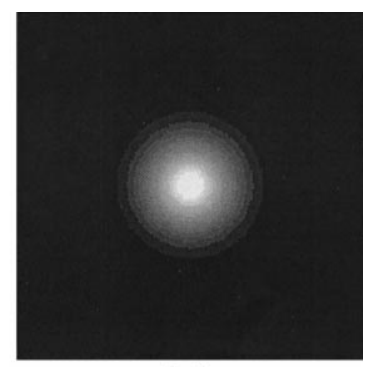

$\mathrm{t}=1$

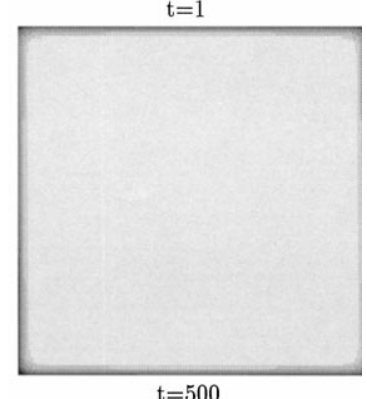

Fig. 1. Open BEC of Rb: Time evolution of $|\phi|^{2}$ using Eq. (20) with $c_{1}=0.52, c_{2}=2.05$ and $\varepsilon=0.5$. The time step is $\left(\Delta_{t}=\right.$ $0.01)$. The initial condition is a Gaussian. The figures are coded using a grey scale (white corresponds to the maximum value of $\left.|\phi|^{2}\right)$.

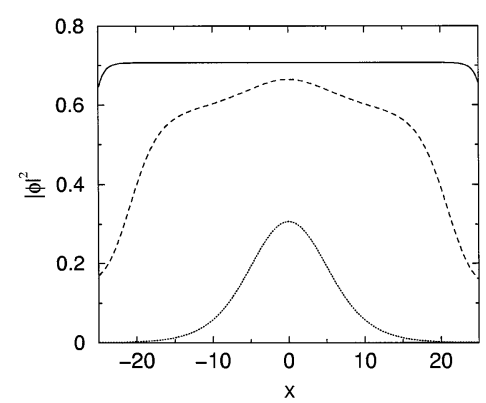

Fig. 2. Cuts of $|\phi|^{2}$ at $y=0$, same parameters as for Fig. 1. The dotted line corresponds to $t=1$, the dashed line is for $t=10$ and the solid line $(t=500)$ corresponds to the final stationary state.

in time with finite difference in space. The chosen boundary conditions (at $x= \pm L_{x} / 2$ and $y=$ $\left.\pm L_{y} / 2\right)$ are

$\frac{\partial \phi}{\partial n}=-0.1 \phi$

where $n$ is the normal at the boundary. Eq. (29) expresses the condition of an isotropic output flux of the condensed BEC (in the ideal situation of zerogravity). The numerical coefficient on the right hand side of Eq. (29) is the dimensionless ratio between $\gamma_{c}$ and the velocity modulus of the condensed atoms, easily evaluated from the ground state solutions of the harmonic oscillator [2]. In fact the ground state of the condensate is not that of the harmonic oscillator, because of the nonlinear term, but for the sake of the computation this is a fair approximation. In Fig. 1 four snapshots of $|\phi(x, y, t)|^{2}$ are shown at different times for the $\mathrm{Rb}$ case $\left(c_{1}=0.52, c_{2}=2.05\right.$ and $\varepsilon=0.5)$. The initial distribution evolves towards a stable quasi-uniform state. Fig. 2 displays three cross section of Fig. 1 at different times, the solid line corresponds to the final stationary state and we observe the nearly uniform condensate on the overall domain. For a uniform pump $R$, the balance between source (uncondensed atom contribution) and sink (boundary escape) eventually yields a uniform condensate profile far from threshold.

Fig. 3 illustrates a quite different situation: The values are now $c_{1}=0.4, c_{2}=-5.1$ and $\varepsilon=0.5$ which corresponds to a $\mathrm{Li}^{*}$ open $\mathrm{BEC}$ in the unstable region of use. The space-time chaotic dynamics emerges after a short transient $(t<10)$. Fig. 4 confirms that $|\phi|^{2}$ is no longer symmetric with respect 


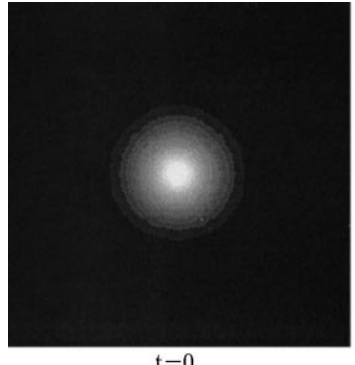

$\mathrm{t}=0$

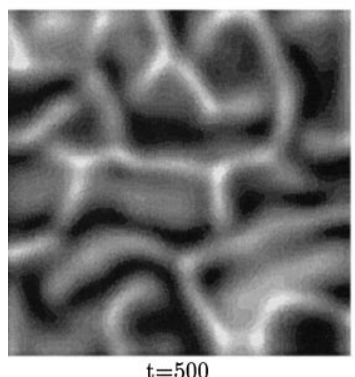

$\mathrm{t}=500$

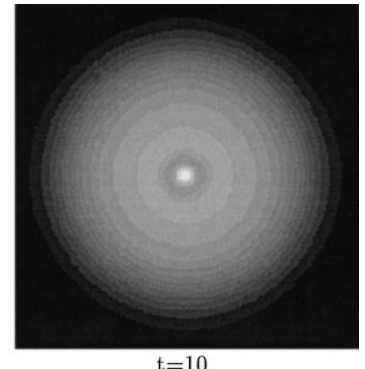

$\mathrm{t}=10$

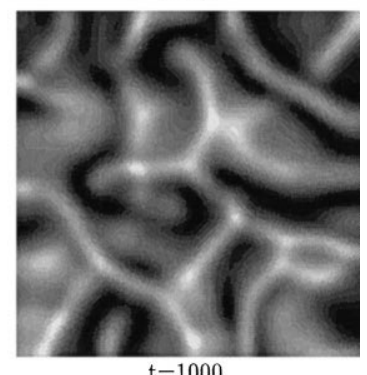

$\mathrm{t}=1000$

Fig. 3. Open BEC of $\mathrm{Li}^{*}$ (escape parameters adjusted to be in the unstable regime): Time evolution of $|\phi|^{2}$ using Eq. (20) with $c_{1}=0.4, c_{2}=-5.1$ and $\varepsilon=0.5$.

to $x=0$ (the same holds for the $y$-axis). Fig. 5 is aimed to show the spatial decorrelation of the signal when the condensate has entered the chaotic regime.

The 1D spatial power spectrum of the $|\phi(x)|^{2}$ function is shown in the lower curve of Fig. 5., the upper curve (solid line) is calculated by averaging the power spectrum of the function $|\phi(x)|^{2}$ over a time interval from $t=500$ until $t=1000$ (taking a sampling time $\delta t=10$ ) within which the dynamics is statistically stationary. The results clearly indicates the large spatial decorrelation of the signal inside the

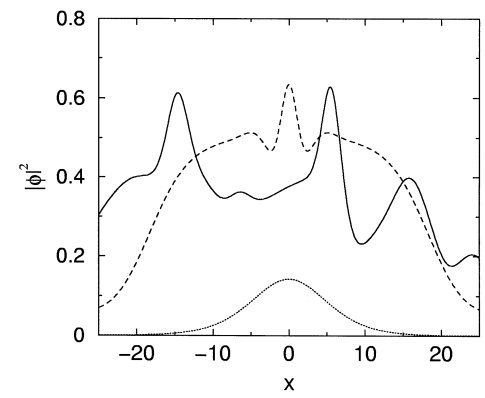

Fig. 4. Cuts of $|\phi|^{2}$ at $y=0$, same parameters as for Fig. 3. The dotted line corresponds to $t=1$, the dashed line is for $t=10$ and the solid line $(t=500)$ corresponds to a chaotic state.

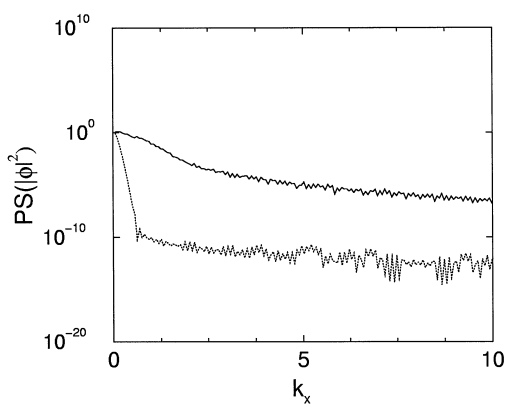

Fig. 5. Spatial power spectrum of $|\phi(x, y=0, t)|^{2}$ for $\mathrm{Li}^{*}$. The lower curve (dotted line) corresponds to $t=1$. The upper curve (solid line) is calculated by averaging the spatial power spectrum of $|\phi(x, y=0, t)|^{2}$ between $t=500$ and $t=1000$ (by steps of $\delta t=10)$.

chaotic regime. Indeed, it is well known that the Fourier transform of a Gaussian function $G \propto$ $\mathrm{e}^{-x^{2} / \sigma_{x}^{2}}$ is again a Gaussian function $\tilde{G} \propto \mathrm{e}^{-k^{2} / \sigma_{k}^{2}}$ with $\sigma_{k} \sigma_{x}=1$. On Fig. 5 it appears that the bandwidth in the Fourier space is much larger in the chaotic regime than for the initial distribution, which means a decorrelation of $|\phi(x)|^{2}$ once the system becomes chaotic.

To give a quantitative feeling, in the case of $\mathrm{Li}^{*}$, we have reduced $\gamma_{c}$ by a factor 10 , which means that the normalization length is increased by $\sqrt{10}$ with respect to Eq. (23), and it is $l_{o}^{*} \approx 10 \mu \mathrm{m}$. In the numerical calculations we have considered a trap of linear size $L=50 l_{o}^{*}$. Since the ratio of the spectral widths between the chaotic and the initial spectra is about 4 (estimated from Fig. 5), it results that the coherence length $\sigma_{c}$ in space time chaos is $\sim 1 / 4$ the length of the initial Gaussian packet $\sigma_{0}$. As seen from Fig. $4 \sigma_{0} \cong 5 l_{o}^{*} \cong 1 / 10 L$, hence $\sigma_{c} \cong 1.2 l_{o}^{*} \cong$ $1 / 40 \mathrm{~L}$. These numerical estimates agree with a densitometric analysis of Fig. 3.

\section{Discussion and conclusions}

Kagan et al. [22] have discussed the collapse of a BEC in ${ }^{7} \mathrm{Li}$ for a number of condensed atoms $N_{0}$ larger than the critical value $N_{\text {crit }}$, that is,

$N_{\text {crit }} \approx \frac{a_{\mathrm{ho}}}{\left|a_{s}\right|}$.

This relation for $N_{\text {crit }}$ is obtained by equating the level spacing $\hbar \omega$ in the trap to the interparticle 
interaction energy $n_{0}|g|=N / V|g|$ where $g=$ $4 \pi \hbar^{2} a_{s} / m$ and $V \approx a_{\mathrm{ho}}^{3}$. Ref. [22] stabilizes the GP via a dissipative term corresponding to 3-body recombination processes. This amounts to a correction corresponding to a 6 th power term in $\phi$ in a free energy potential. The dissipative equation of Ref. [22] is then

$$
\begin{aligned}
\dot{\phi}= & -\mathrm{i}[G P]-\xi|\phi|^{4} \phi \\
& + \text { (pumping from the uncondensed portion }) .
\end{aligned}
$$

In Eq. (20), we have already treated the last term, here expressed in words, by the Kneer et al. approach [9]. Let us now compare the 5th power real damping entering Eq. (31) with the 3 th power real damping of Eq. (20). The cubic term is of the form $G_{3} \phi$, where

$$
G_{3}=\frac{R \Gamma^{2}}{2 \gamma_{u}^{2}}|\phi|^{2}=\frac{\gamma_{c} \Gamma}{\gamma_{u}}|\phi|^{2} .
$$

Using the numerical values corresponding to the ${ }^{87} \mathrm{Rb}$ we obtain $G_{3} \approx 48 \mathrm{~s}^{-1}$, for ${ }^{7} \mathrm{Li}$ we have $G_{3} \approx$ $1920 \mathrm{~s}^{-1}$. The cubic rate $\left(G_{3}\right)$ is a combination of the three characteristic rates of an open BEC. In a similar way we can introduce the rate $G_{5}=\xi|\phi|^{4}$. Taking the numerical values provided in Ref. [22] we have the following ratios between the two dissipation rates

$$
\frac{G_{3}}{G_{5}}= \begin{cases}480 & \text { for }{ }^{87} \mathrm{Rb} \\ 2 \times 10^{4} & \text { for }{ }^{7} \mathrm{Li}\end{cases}
$$

This result clearly indicates that for an open BEC, the 3-body recombination is negligible with respect to the saturation cubic term that comes from the coupling between the condensed and uncondensed phase of the open BEC.

To summarize, in this paper we have shown that in the framework of an atom-laser approach via two coupled equations, one for the uncondensed phase and the other one for the condensed phase, addition of a diffusion term for the uncondensed atoms and application of a proper adiabatic elimination procedure leads to a CGL dynamical equation for an open BEC. In the case of negative scattering length, a suitable adjustment of the escape rate implies entering the unstable regime of the CGL dynamics. Furthermore, within the chosen ranges of the parameters $\left(\varepsilon, c_{1}, c_{2}\right)$, the 3 -body recombination processes have a negligible influence.

\section{Note added in proof}

Notice that the Benjamin-Feir instability means generically that the $k=0$ (long wavelength) perturbation modes is unstable [10]. The dissipative case discussed by us, leading to space-time chaos, is within the framework of Refs. [11,12]; however the initial phenomenon is a modulational instability without chaos, as discussed for the case of two interacting Bose condensates in Ref. [23].

\section{Acknowledgements}

The authors acknowledge E. Arimondo, T. Hansch, L. Pitaevskii and the BEC group at LENS Firenze for fruitful discussions. J.B. was partially supported by a EU Network grant (FMRXCT960010) 'Nonlinear dynamics and statistical physics of spatially extended systems' and by the Belgian Programme on Interuniversity Poles of Attraction (PAI 04-6) initiated by the Belgian State Federal Office of Scientific, Technical and Cultural Affairs. This work was partially supported by INFM through the Advanced Research Project CAT.

\section{References}

[1] E.P. Gross, Nuovo Cimento 20 (1961) 454; L.P. Pitaevskii, Zh. Eksp. Teor. Fiz. 40 (1961) 646 [Sov. Phys. JETP 13 (1961) 451].

[2] F. Dalfovo, S. Giorgini, L.P. Pitaevskii, S. Stringari, Rev. Mod. Phys. 71 (1999) 463.

[3] M.H. Anderson, J.R. Ensher, M.R. Matthews, C.E. Wieman, E.A. Cornell, Science 269 (1995) 198.

[4] C.C. Bradley, C.A. Sackett, J.J. Tollett, R.G. Hulet, Phys. Rev. Lett. 75 (1995) 1687.

[5] K.B. Davis, M.O. Mewes, M.R. Andrews, N.J. van Druten, D.S. Durfee, D.M. Kurn, W. Ketterle, Phys. Rev. Lett. 75 (1995) 3969.

[6] M.O. Mewes, M.R. Andrews, D.M. Kurn, D.S. Durfee, C.G. Townsend, W. Ketterle, Phys. Rev. Lett. 78 (1997) 852.

[7] E.W. Hagley, L. Deng, M. Kozuma, J. Wen, K. Helmerson, S.L. Rollston, W.D. Phillips, Science 283 (1999) 1706.

[8] I. Bloch, T.W. Hansch, T. Esslinger, Phys. Rev. Lett. 82 (1999) 3003. 
[9] B. Kneer, T. Wong, K. Vogel, W.P. Schleich, D.F. Walls, Phys. Rev. A 58 (1998) 4841.

[10] T.B., Benjamin, J.E. Feir, J. Fluid Mech. 27 (1967) 417.

[11] B. Shraiman et al., Physica (Amsterdam) D 57 (1992) 241; H. Chaté, Nonlinearity 7 (1994) 185; H. Chaté, in: P.E. Cladis, P. Palffy-Muhoray (Eds.), Spatiotemporal Patterns in Nonequilibrium Complex Systems, Addison-Wesley, New York, 1995.

[12] H. Chate, P. Manneville, Physica A 224 (1996) 348.

[13] R. Dum, J.I. Cirac, M. Lewenstein, P. Zoller, Phys. Rev. Lett. 80 (1998) 2972.

[14] R.A. Sammut, A.V. Buryak, Yu.S. Kivshar, J. Opt. Soc. Am. B 15 (1998) 1488.
[15] A. Hasegawaand, F. Tappert, Appl. Phys. Lett. 23 (1973) 142.

[16] A. Kumar, Phys. Rep. 187 (1990) 63.

[17] R.J.C. Spreeuw, T. Pfau, U. Janicke, M. Wilkens, Europhys. Lett. 32 (1995) 469.

[18] R. Kubo, J. Phys. Soc. Jpn. 12 (1957) 570.

[19] T. Callen, T.A. Welton, Phys. Rev. 83 (1951) 34.

[20] H. Haken, Advanced Synergetics, Springer, Berlin, 1983.

[21] BEC group at LENS, Firenze, private communication, 1999.

[22] Y. Kagan, A.E. Muryshev, G.V. Shlyapnikov, Phys. Rev. Lett. 81 (1998) 993.

[23] S. Metens, D. Lima, P. Borckmans, G. Dewel, Fringe patterns of Bose condensate, cond-mat/9807399. 